\title{
Mechanical Properties of Self-Assembled Microtubule Curved Protofilaments
}

\author{
Ramana Pidaparti ${ }^{\circledR}$, Divya Jakkam \\ School of Environmental, Civil, Agriculture and Mechanical Engineering College of Engineering, University \\ of Georgia, Athens, GA, USA
}

Correspondence to: Ramana Pidaparti,rmparti@uga.edu

Keywords: Microtubule, Protofilament, Tubulin, Finite Element Method, Mechanical Properties

Received: February 18, $2020 \quad$ Accepted: March 28, $2020 \quad$ Published: March 31, 2020

Copyright $\odot 2020$ by author(s) and Scientific Research Publishing Inc.

This work is licensed under the Creative Commons Attribution International License (CC BY 4.0).

http://creativecommons.org/licenses/by/4.0/

\section{(c) (i) Open Access}

\section{ABSTRACT}

The microtubule self-assembly process involves the basic building blocks, alpha and beta tubulins which spontaneously bind to one another through polymerization and under controlled intracellular conditions form protofilaments which in turn assemble into microtubules. The mechanical properties of the self-assembled protofilaments play an important role in formation of the microtubules. In this study, we investigate the mechanical properties of the experimentally self-assembled protofilaments (straight and curved) for under different loadings through 3D finite element analysis. Results of force-deformation and stiffness values obtained from the finite element analysis are presented. The results indicate that the stiffness and maximum stress properties change with varying protofilamant curvature. These force-deformation behaviors and stress distributions should help further understand the contribution of protofilaments mechanical properties in forming self-assembled microtubules.

\section{INTRODUCTION}

Microtubules (MT) can be found in all eukaryotic cells. Microtubules are filamentous intracellular structures ( $25 \mathrm{~nm}$ in diameter and quite long) that are responsible for various kinds of movements and are responsible for cell division, organization of intracellular structure, and intracellular transport, as well as ciliary and flagellar motility [1-3]. The MT self-assembly process involves the basic building blocks, "tubulin monomers" (alpha and beta) with slightly different properties. Both alpha and beta tubulins spontaneously bind one another through polymerization to form a function subunit called a "hetrodimer". These tubulin hetrodimers assemble into linear "protofilaments" under controlled intracellular conditions. Protofilaments in turn assemble into microtubules. Within microtubules, individual tubulins may exist in different states, which can change on various time scales, thus exhibiting different properties. 
Janosi et al. [4] observed from their study of microtubules with electron micrographs, that they were made of 10 to 16 protofilaments, with either 13 or 14 protofilaments in $90 \%$ of the cases in their study. They also noted that slight bending of the protofilament at the ends allows the release of some built-in stress. They observed that the change in the lateral bond strength between protofilaments plays a role in the polymerizing and deploymerizing of the MTs and also, that there is a particular correlation between protofilament number and curvature at the MT tips [5].

Kis et al. [6] investigated the anisotropic properties of MTs by using an AFM (Atomic force microscope) on a perforated surface. They believed that the protofilaments of an MT are analogous to ropes of single walled carbon nanotubes and determined a relation between the Young's modulus and shear modulus. Experiments were conducted by Shi et al. [7] on a single microtubule where they observed the link between flexural rigidity and the MT length using a laser trapping technique and dark-field microscopy. An atomic resolution model of an MT was built to determine stress-strain dependence on extension and compression, thereby obtaining values of the Young's modulus which was comparable to previously published data [8]. A coarse-grained model of the MT was developed to study the mechanical properties of the MTs by assuming different types of lateral bonding between adjacent protofilaments [9]. Donhauser et al. developed a finite element model to evaluate the effect of radial deformation on various MT types and found that the orientation of the protofilament does not have any effect on the radial stiffness of the MT [10].

The mechanics of individual MTs have been extensively studied, specifically the molecular mechanisms controlling microtubule rigidity. Motamedi and Mashhadi [11] studied the bond interactions within each of the tubulin dimers using Molecular Dynamics (MD) simulations, and determined the Young's modulus and other mechanical properties of the MTs using a finite element model. They assumed each tubulin monomer to be a sphere with a nonlinear spring connecting them to achieve their results. Kasas et al. used a finite element model [12] assuming tubulin dimensions to determine the mechanical properties. $\alpha$ - and $\beta$-tubulins were assumed to be the same, structurally for their model. Civalek and Demir [13] proposed a nonlocal finite element model based on the Euler-Bernoulli beam model to analyze the effect of buckling on MTs. They applied the Winkler spring model to the elastic matrix surrounding the microtubule and noticed an increase in the buckling loads with an increase in the elastic matrix parameter. Shahinnejad et al. [14] developed a 3D finite element model and investigated the mechanical properties of axonal microtubule bundles by studying the strain-stiffening behavior of these bundles under uniaxial tension and torsional loading. Kim et al. [15] developed a finite element model of straight protofilaments and investigated the effects of their mechanical behavior under different loadings. They found that protofilament behaves non-linearly under tension and torsion but linearly under bending. Due to the important role played by curved protofilaments in MT self-assembly process, it is important to further investigate their mechanical behavior under multiple loadings.

In this study, we investigated the mechanical properties of the experimentally self-assembled protofilaments (straight and curved) for through 3D finite element analysis. After creating the protofilaments geometry and assigning the properties, multiple analyses were carried out under tension, bending and torsional loadings. The results of force-deformation and stiffness/stress distributions obtained from the finite element analysis are presented and discussed.

\section{COMPUTATIONAL ANALYSIS}

\subsection{Geometry and Dimensions}

The basic unit of a microtubule is the tubulin dimer which bonds longitudinally to other dimers thereby forming protofilaments. We assume the tubulin monomer to be spherical in shape for developing the computational analysis using 3D finite element method. Chr'etien et al. [16] observed that the number of protofilaments varied from twelve to seventeen, with sixteen and seventeen protofilaments being in the minority and thirteen being the majority population. In our study, we developed a protofilament model 
consisting of 12 tubulin units modeled as spheres of diameter $4.05 \mu \mathrm{m}$ and the entire protofilament measured up to $48.6 \mu \mathrm{m}$ with a volume of $417.42 \mu \mathrm{m}^{3}$. The tubulins are connected to each other by means of a beam of circular cross-section of radius $0.2025 \mu \mathrm{m}$. The beam material is the same as that of the tubulin material. All the tubulins are bonded to each other and any sliding or separation between edges of the spheres is not allowed.

The geometry for the protofilament is modeled in the ANSYS Design Modeler software in which 2D geometry can be created, edited and converted to $3 \mathrm{D}$ models. It is assumed that the protofilament is fixed at one end, while free at the other end, where the load is applied. Figure 1 shows the protofilament model considered for computations in this study.

Based on the experimental observations [17], self-assembled curved protofilaments were grouped into low and high curvature as shown in Figure 2. For finite element analysis, it is assumed that all tubulins have a Young's modulus of $1.85 \mathrm{GPa}$ and a poission's ratio of 0.3 . Three different loadings were considered, which include tensile load (force varying between $0.01 \mu \mathrm{N}$ and $15 \mu \mathrm{N}$ ), bending (force varying between $0.01 \mu \mathrm{N}$ and $10 \mu \mathrm{N}$ ) and torsion (torsional moment varying between $0.01 \mu \mathrm{N} \cdot \mu \mathrm{m}$ and $20 \mu \mathrm{N} \cdot \mu \mathrm{m}$ ). These configurations were analyzed under different loadings to estimate their mechanical behavior. All simulations were performed using the software ANSYS 19.1 (ANSYS Inc., Canonsburg, PA, USA) Mechanical package.

\subsection{Convergence Study and Validation}

A mesh convergence was carried to determine the level of elements required for accurate deformation and stress results. We used the proximity and curvature size function to generate the mesh for the model, with a minimum element size of $0.4 \mu \mathrm{m}$. The smaller the element size for the mesh, the higher is the accuracy of the results. This was confirmed by conducting a mesh convergence study: first for a cantilever beam, and then for the straight protofilament model as shown in Figure 3. The number of finite elements varied from 3000 to 56,000 tetrahedral elements. The converged mesh includes about 56,198 elements which were used in obtaining the results.

In order to validate our 3D finite element analysis results, a beam element solution was conducted under tension and bending loadings for the model shown in Figure 3. The results of bending deflection at the free end obtained from beam model were compared to those obtained from analytical solution and a good agreement was found. The tip defection under tensile load obtained from analytical solution 25.946 $\mu \mathrm{m}$ closely matches to those obtained from finite element analysis of $25.839 \mu \mathrm{m}$. Similarly, the bending defection obtained from analytical solution $0.1494 \mathrm{~m}$ closely matches to those obtained from finite element analysis of $0.1491 \mathrm{~m}$. Overall, the results of tip deflections at the free end obtained from beam model compares well with those obtained from analytical solution, thus validating the finite element model in this study.

\section{RESULTS AND DISCUSSION}

\subsection{Load - Deformation Behavior}

The force-displacement responses from the computational simulations under tensile, bending and torsion are presented in Figures 4-6, respectively. It can be seen in Figure 4 that the deformation due to

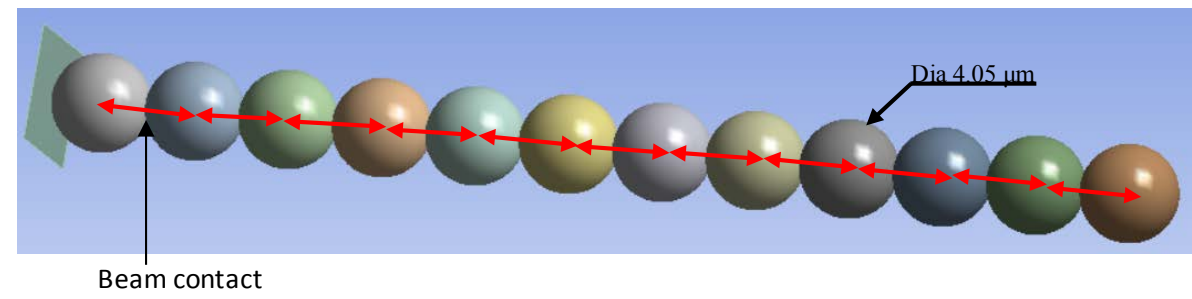

Figure 1. Protofilament model considered for computational analysis. 


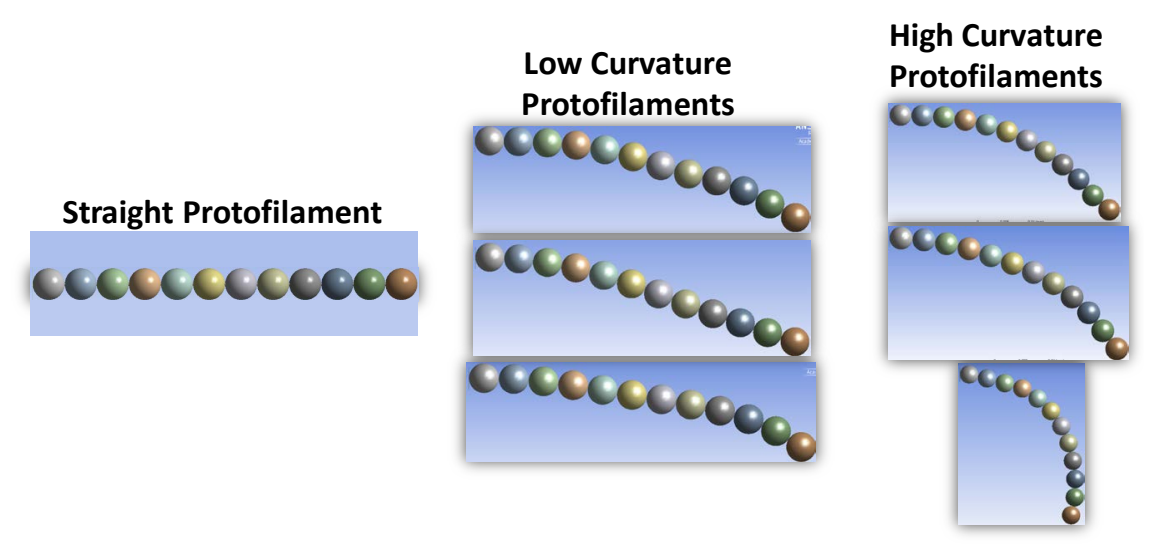

Figure 2. Curved protofilament models considered for computational analysis.

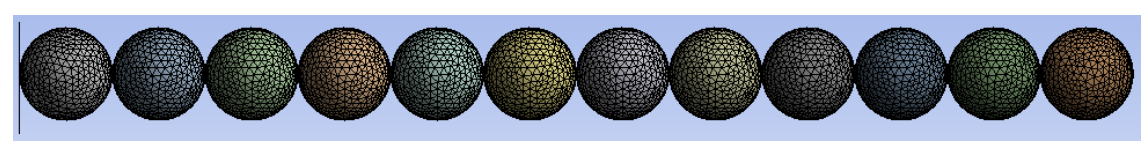

FEA model

Figure 3. Finite element model for straight protofilament considered.

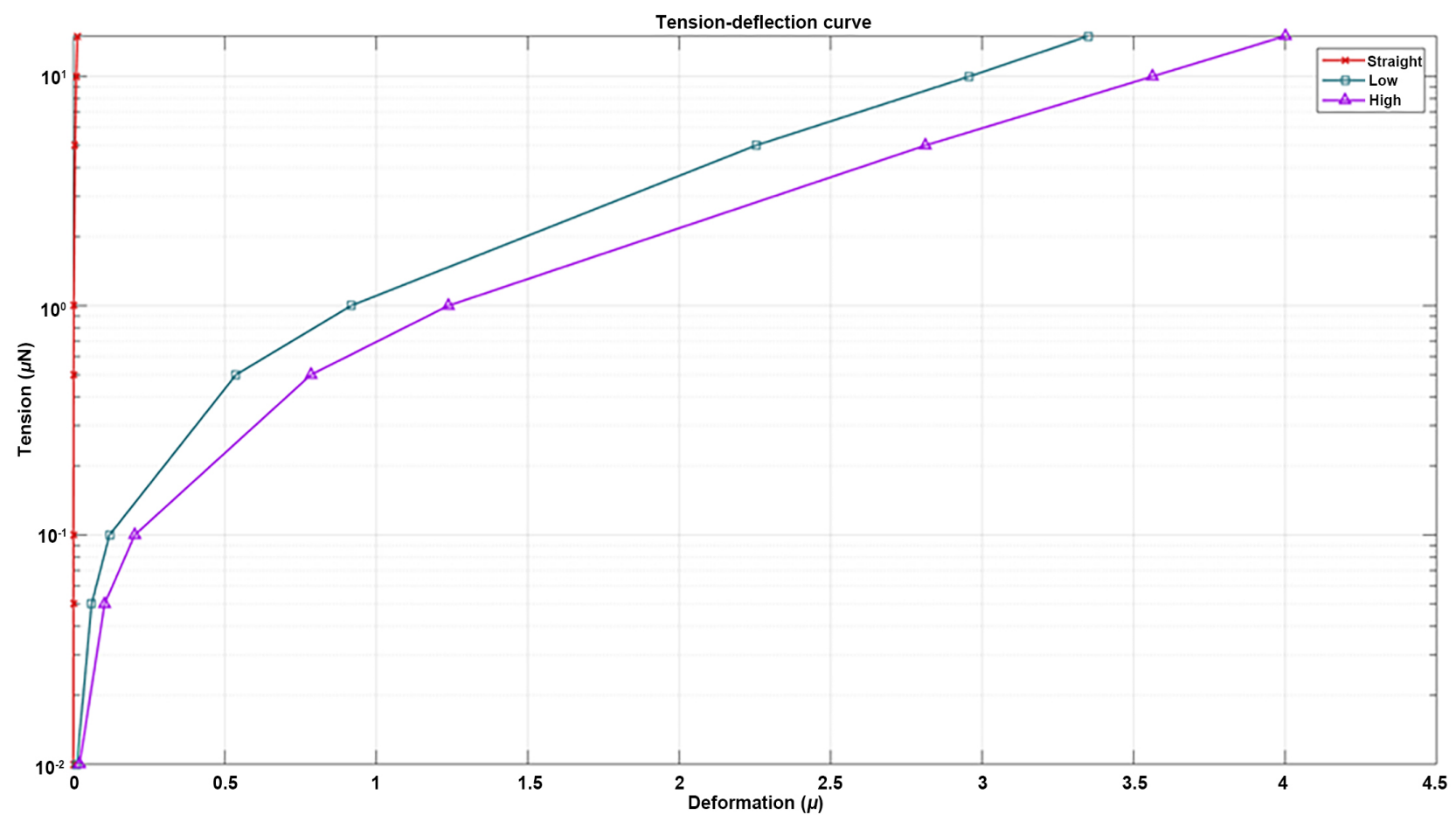

Figure 4. Load-deformation behavior of curved protofilaments under tensile loading.

tension for the straight protofilament configuration is the least in comparison to curved protofilaments. For the two curved (low and high) protofilament configurations considered, low curvature protofilaments exhibits stiffer behavior than high curvature protofilaments. Also, the deformation is higher for high curvature protofilaments in comparison to low curvature protofilaments. There is an inverse relation between the slope of the force-deformation curves and the rate of change in the deformation, which means that as 
the slope of the protofilament configuration decreases, the deformation due to tension also increases non-linearly.

It can be seen from Figure 5 that the deformation due to bending varies from high, straight and low curvature protofilament configurations, especially at higher loads. Also, it can be observed that at small bending loads, the initial deformation is almost about the same for all three protofilaments configurations. All three protofilament configurations follow a similar non-linear behavior.

The torsional load deformation behavior presented in Figure 6 shows that with increasing protofilament curvature, the deformation increases. It can be seen from Figure 6 that at small torsional loads, the initial deformation is almost about the same for all three protofilaments configurations, and then changes with increasing load. Also, it can be observed that all three protofilament configurations exhibit non-linear behavior and vary with increasing loads, especially at higher torsional loads. The force-deformation behavior trend under bending and torsion for straight protofilament qualitatively matches to those obtained by Kim et al. [15]. Overall, it can be seen from Figures 4-6 that curved protofilaments exhibit a different non-linear behavior under different loads, leading to different mechanical behavior.

\subsection{Stress Distributions}

The von-Mises stress distributions for the three protofilament configurations (straight, low and high curvatures) under tensile, bending and torsional loadings are presented in Figure 7. It can be seen in Figure 7 that the stress magnitudes and distributions vary considerably with protofilament curvatures. Also, the stress results vary with the applied loadings, especially the interface stresses between tubulins for both straight and curved protofilaments. In order to estimate the maximum stresses under different loadings, Figure 8 shows how the curvature affects the stresses under different loadings.

It can be seen from Figure $\mathbf{8}$ that the stresses are higher in tension for straight protofilament compared to curved protofilaments. For bending loading, high curvature protofilament stress is lower than straight protofilament. Similarly, under torsional loading, low curvature protofilament configuration has high stress. Overall the results presented in Figure 8 demonstrate that both curvature and loading greatly affect the mechanical behavior of protofilaments.

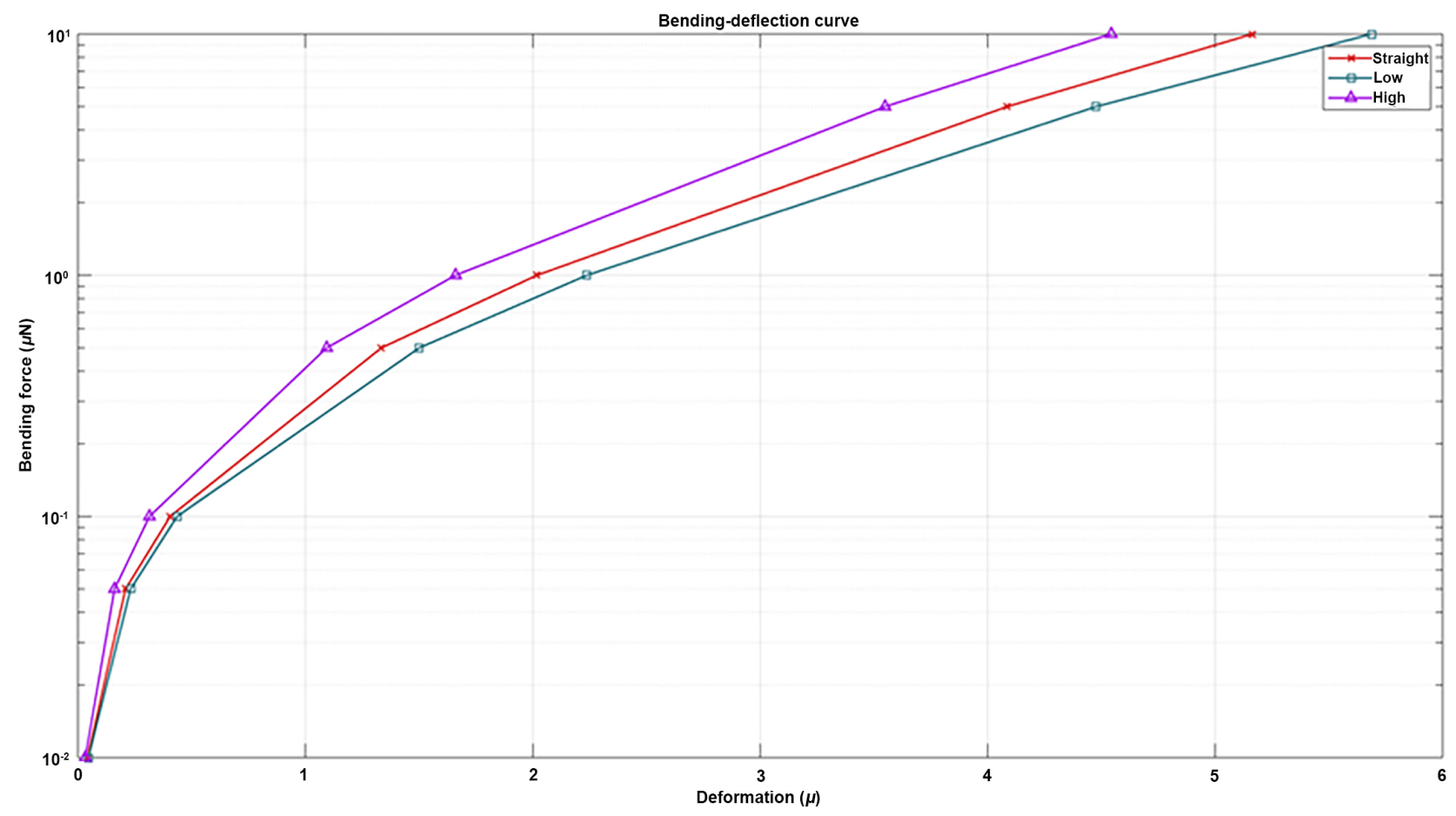

Figure 5. Load-deformation behavior of curved protofilaments under bending. 


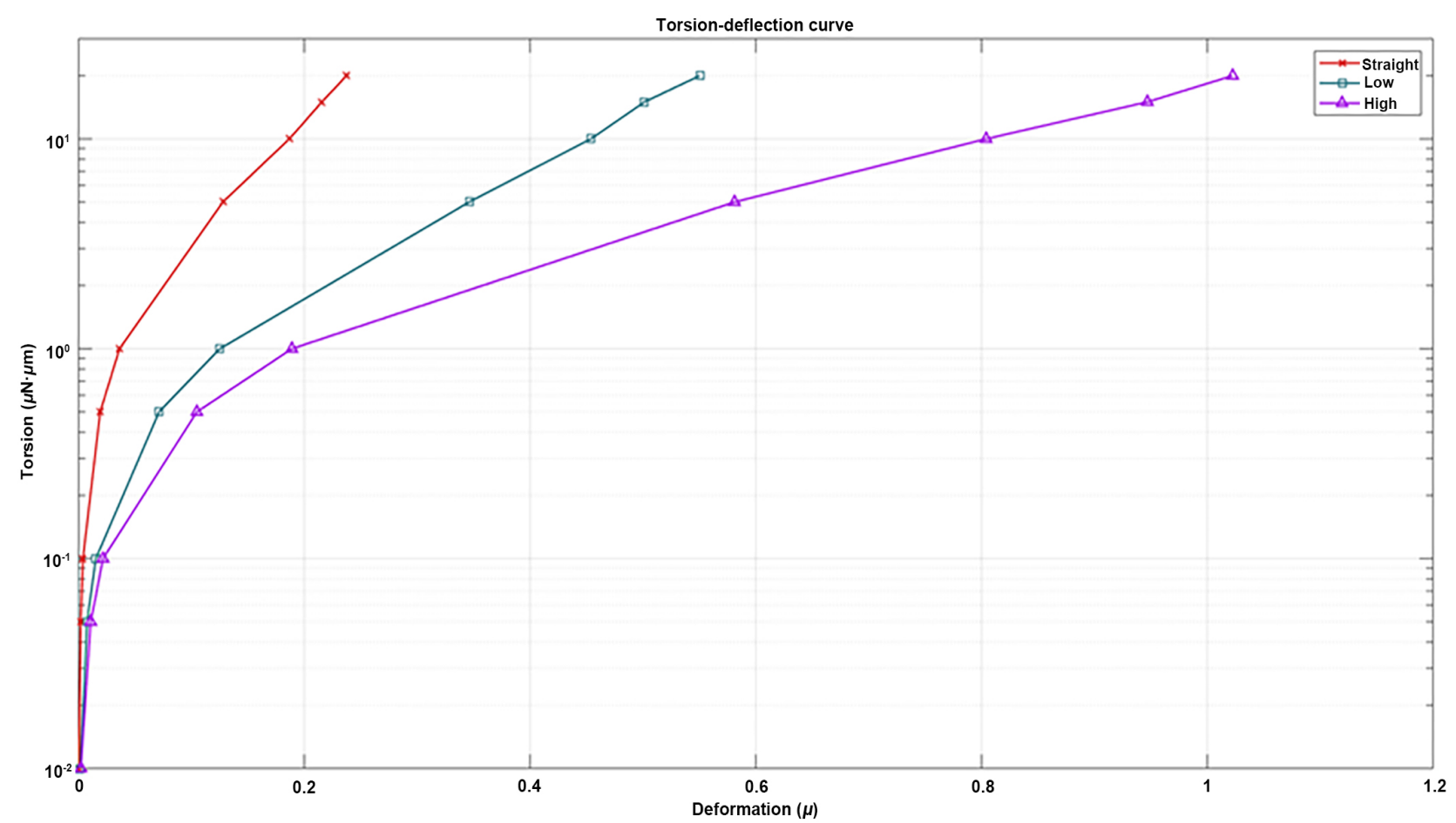

Figure 6. Load-deformation behavior of curved protofilaments under torsion.

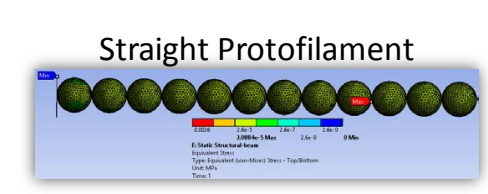

Straight Protofilament

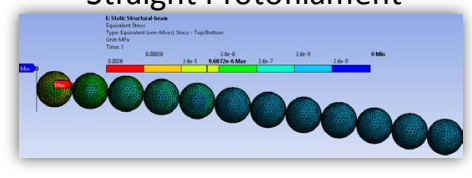

Straight Protofilament

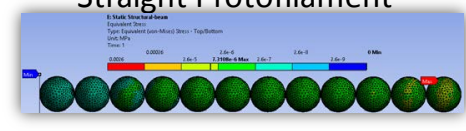

Low Curvature Protofilament

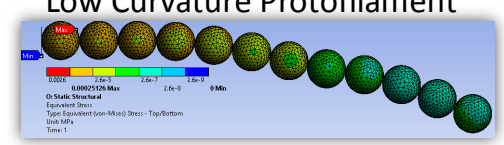

(a) Tension Loading

Low Curvature Protofilament

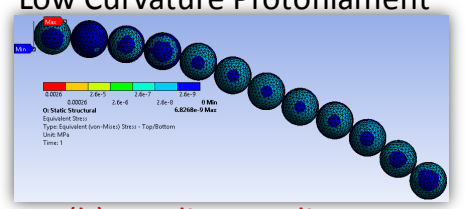

(b) Bending Loading

Low Curvature Protofilament

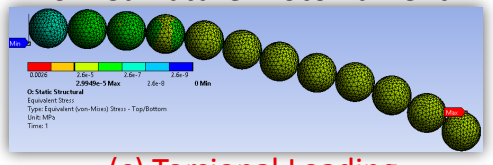

(c) Torsional Loading

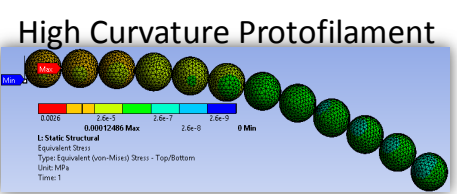

High Curvature Protofilament

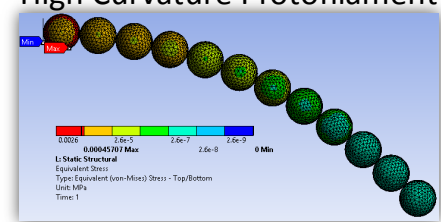

High Curvature Protofilament

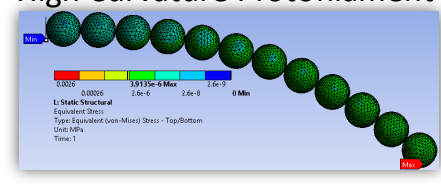

Figure 7. Von-Mises stress distributions for straight and curved protofilaments under different loadings.

\section{CONCLUSIONS}

In this study, we investigated the mechanical properties of the experimentally self-assembled protofilaments (straight and curved) under different loadings through 3D finite element analysis. An investigation of straight and curved protofilaments with $\alpha$ and $\beta$ tubulins as building blocks of microtubules was carried out to estimate their mechanical behavior under different loadings. Geometric models were built from experimentally self-assembled protofilaments (straight and curved) and analyzed using a 3D finite element analysis. Results of load-deformation, stiffness and stress values obtained from the finite element 


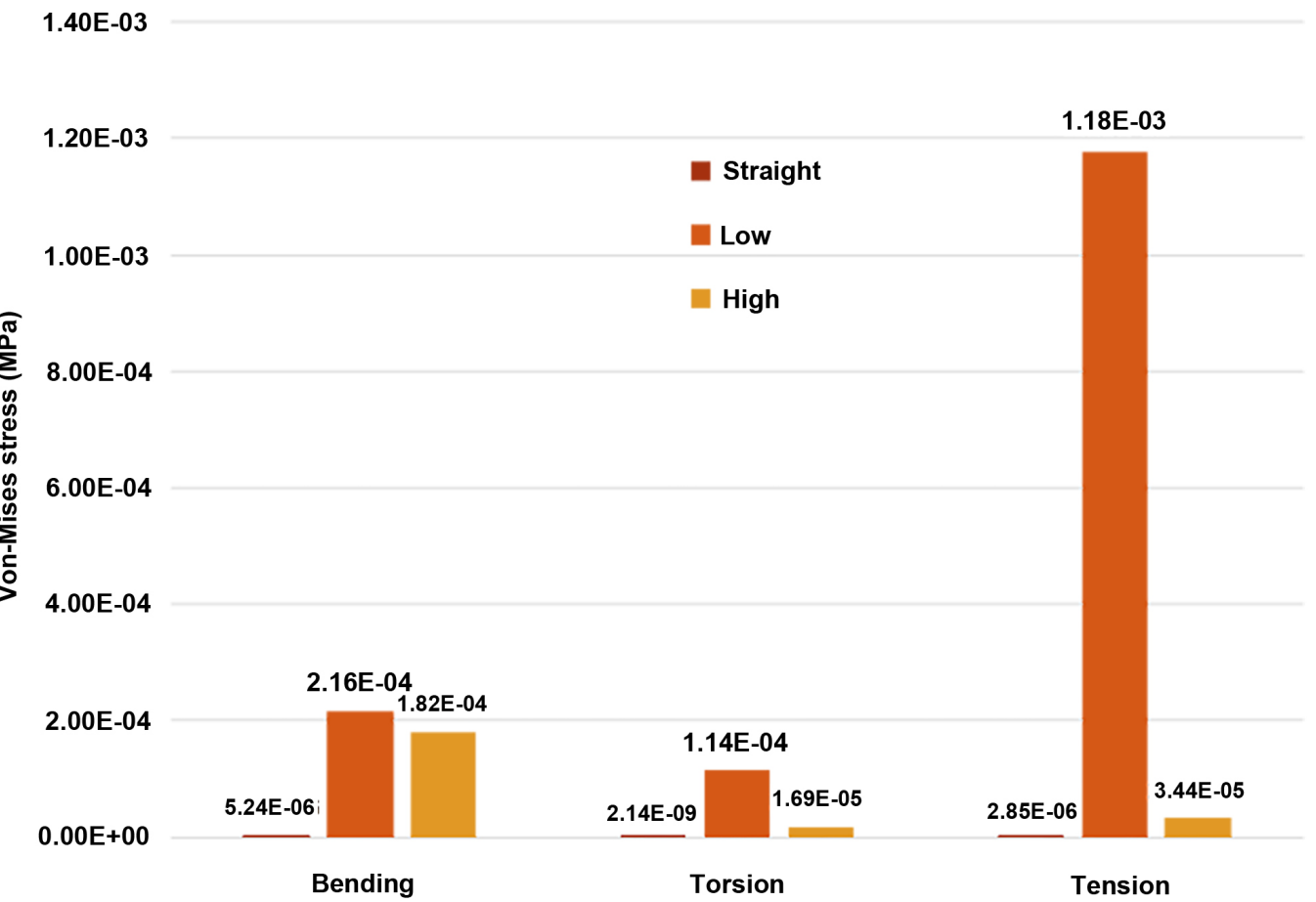

Figure 8. Maximum von-Mises stresses for curved protofilaments under different loadings.

analysis are presented. The results indicate that both load-deformation behavior and stress distributions vary non-linearly with increasing protofilamant curvature and also with applied loadings. These findings should help further understand the contribution of protofilaments mechanical properties in forming self-assembled microtubules.

\section{ACKNOWLEDGEMENTS}

The authors thank Dr. Jong Won Kim for the helpful discussions and the model development. The first author thanks the NSF for supporting this work through a grant CDS\&E-1610812.

\section{CONFLICTS OF INTEREST}

The authors declare no conflicts of interest regarding the publication of this paper.

\section{REFERENCES}

1. Alberts, B. (2017) Molecular Biology of the Cell. Garland Science, New York.

2. Dogterom, M. and Surrey, T. (2013) Microtubule Organization in Vitro. Current Opinion in Cell Biology, 25, 23-29. https://doi.org/10.1016/j.ceb.2012.12.002

3. Walczak, C.E., Cai, S. and Khodjakov, A. (2010) Mechanisms of Chromosome Behaviour during Mitosis. Nature Reviews Molecular Cell Biology, 11, 91-102. https://doi.org/10.1038/nrm2832

4. J'anosi, I.M., Chretien, D. and Flyvbjerg, H. (2002) Structural Microtubule Cap: Stability, Catastrophe, Rescue, and Third State. Biophysical Journal, 83, 1317-1330. https://doi.org/10.1016/S0006-3495(02)73902-7

5. Jnosi, I.M., Chrtien, D. and Flyvbjerg, H. (1998) Modeling Elastic Properties of Microtubule Tips and Walls. European Biophysics Journal, 27, 501-513. https://doi.org/10.1007/s002490050160 
6. Kis, A., Kasas, S., Babi'c, B., Kulik, A.J., Benoit, W., Briggs, G.A.D., Schönenberger, C., Catsicas, S. and Forr'o, L. (2002) Nanomechanics of Microtubules. Physical Review Letters, 89, Article ID: 248101. https://doi.org/10.1103/PhysRevLett.89.248101

7. Yj, S. (2008) Relevance of Timoshenko-Beam Model to Microtubules of Low Shear Modulus. Physica, $41,213$. https://doi.org/10.1016/j.physe.2008.06.025

8. Wells, D.B. and Aksimentiev, A. (2010) Mechanical Properties of a Complete Microtubule Revealed through Molecular Dynamics Simulation. Biophysical Journal, 99, 629-637. https://doi.org/10.1016/j.bpj.2010.04.038

9. Wu, Z., Nogales, E. and Xing, J. (2012) Comparative Studies of Microtubule Mechanics with Two Competing Models Suggest Functional Roles of Alternative Tubulin Lateral Interactions. Biophysical Journal, 102, 2687-2696. https://doi.org/10.1016/j.bpj.2012.05.003

10. Donhauser, Z.J., Jobs, W.B. and Binka, E.C. (2010) Mechanics of Microtubules: Effects of Protofilament Orientation. Biophysical Journal, 99, 1668-1675. https://doi.org/10.1016/j.bpj.2010.06.065

11. Motamedi, M. and Mashhadi, M.M. (2016) Dynamic Simulation and Mechanical Properties of Microtubules. Journal of Solid Mechanics, 8, 781-787.

12. Kasas, S., Kis, A., Riederer, B.M., Forr'o, L., Dietler, G. and Catsicas, S. (2004) Mechanical Properties of Microtubules Explored Using the Finite Elements Method. ChemPhysChem, 5, 252-257. https://doi.org/10.1002/cphc.200300799

13. Civalek, O. and Demir, C. (2016) A Simple Mathematical Model of Microtubules Surrounded by an Elastic Matrix by Nonlocal Finite Element Method. Applied Mathematics and Computation, 289, 335-352. https://doi.org/10.1016/j.amc.2016.05.034

14. Shahinnejad, A., Haghpanahi, M. and Farmanzad, F. (2013) Finite Element Analysis of Axonal Microtubule Bundle under Tension and Torsion. Procedia Engineering, 59, 16-24. https://doi.org/10.1016/j.proeng.2013.05.088

15. Kim, J., Li, N., Pidaparti, R. and Wang, X. (2018) Microtubular Protofilament Analysis Based on Molecular Level Tubulin Interaction. Molecular and Cellular Biomechanics, 15, 127-144.

16. Chretien, D. and Wade, R.H. (1991) New Data on the Microtubule Surface Lattice. Biology of the Cell, 71, 161-174. https://doi.org/10.1016/0248-4900(91)90062-R

17. Jakkam, D. and Thesis, M.S. (2018) A Study on Self-Assemble and Structural Analysis of Protofilaments. University of Georgia, Athens. 